\title{
Up-regulation of miR-146b-3p protects septic mice with acute respiratory distress syndrome by inhibiting PI3K/AKT signaling pathway
}

\author{
Yao Liu ${ }^{1}$ • Jin-Qiang Zhu ${ }^{1} \cdot$ Xiao-Hong Jin ${ }^{1} \cdot$ Mei-Ping Dong ${ }^{1}$ - Jun-Fen Zheng ${ }^{1}$ \\ Received: 26 March 2020 / Accepted: 22 May 2020 / Published online: 2 June 2020 \\ (C) Springer Science+Business Media, LLC, part of Springer Nature 2020
}

\begin{abstract}
This study aimed to explore the role of miR-146b-3p in acute respiratory distress syndrome in septic mice. Ten mice were randomly selected as normal group $(\mathrm{n}=10$, without any treatment) and 60 septic mice with acute respiratory distress syndrome were divided into model group $(n=10$, without any treatment), negative control (NC) mimic group $(n=10$, injected with NC mimic), miR-146b-3p mimic group $(n=10$, injected with miR-146b-3p mimic), si-NC group $(n=10$, injected with PI3K $\gamma$ siRNA NC), si-PI3K $\gamma$ group ( $\mathrm{n}=10$, injected with PI3K $\gamma$ silencing plasmid), and miR-146b-3p mimic + oe-PI3K $\gamma$ group $(\mathrm{n}=10$, injected with miR-146b-3p mimic $+\mathrm{PI} 3 \mathrm{~K} \gamma$ overexpression plasmid). We found that miR-146b-3p negatively regulated PI3K $\gamma$. Compared with normal group, model mice had decreased expression of miR-146b-3p, increased expressions of PI3K $\gamma$, p-AKT, ASC, NLRP3 and Caspase-1 proteins, higher W/D ratio, and more serum IL-1 $\beta$ and IL-18 content (all $P<0.05$ ). All indicators in miR-146b-3p mimic group and si-PI3K $\gamma$ group were significantly improved as compared to model group (all $P<0.05)$. Over-expression of PI3K $\gamma$ could weaken the treatment effect of miR-146b-3p mimic in model mice. Therefore, upregulation of miR-146b-3p can inhibit PI3K/AKT signaling pathway to improve acute respiratory distress syndrome in septic mice.
\end{abstract}

Keywords miR-146b-3p · PI3K/AKT $\cdot$ Sepsis · Acute respiratory distress syndrome

\section{Introduction}

Acute respiratory distress syndrome (ARDS) is a noncardiogenic pulmonary edema caused by the accumulation of extravascular lung water, and it is a major complication of severe sepsis and septic shock (Nystrom 2008). ARDS is characterized by hypoxemia, pulmonary edema, and significant respiratory failure over time, which leads to multiple organ failure and high mortality (up to 60\%) (Wang et al. 2017; Annane et al. 2006; Chawla et al. 2016; Park et al. 2017). Lipopolysaccharide (LPS) is the main agent to construct sepsis-induced ARDS model as LPS can activate inflammatory cells to release inflammatory factors, such as interleukin-1 $\beta$ (IL-1 $\beta$ ) and interleukin-18 (IL-18) (Qi et al. 2016). NLRP3 inflammasome as a protein complex contains

Jun-Fen Zheng

zhengjunfen9n40@163.com

1 Department of Emergency Center, The First People's Hospital of Wenling, No.333 Chuan'an South Road, Chengxi Street, Zhejiang Province 317500 Wenling, China pro-caspase-1 and apoptosis-associated speck-like protein caspase recruitment domain (ASC); active caspase-1 stimulates IL-1 $\beta$ and IL-18, causing the release of these active cytokines ( $\mathrm{Li}$ et al. 2018a). NLRP3 inflammasome plays a vital role in the pathological process of ARDS.

AKT1 gene, known as protein kinase $\mathrm{B} \alpha$, is a key member in AKT family. Moreover, AKT is generally recognized as a key factor in PI3K (phosphatidylinositol 3-kinase) /AKT pathway and acts as a crucial role in cell differentiation, proliferation, metabolism, apoptosis, protein synthesis and transcription (Li et al. 2015). Previous studies have indicated that ARDS can be treated by the inhibition of PI3K/AKT signaling pathway (Ji and Wang 2019; Li et al. 2018b; Yanagi et al. 2015; Zheng et al. 2018).

ARDS is a complex pathological process, which is regulated by transcription factors as well as microRNA (miRNA). miRNA is a small endogenous non-coding RNA molecule that has approximately 18-24 nucleotides. It usually binds to the target messenger RNA in the 3' untranslated region to inhibit its post-transcriptional expression (Wang et al. 2015). Several studies have found that multiple miRNAs affect the occurrence of ARDS. For example, miRNA-122 has relations 
with the mortality of ARDS and acute liver injury (Rahmel et al. 2018). miRNA-211 inhibits the function of macrophages releasing IL-10 in LPS-induced ARDS rats (Wang et al. 2018). Down-regulation of miRNA-494 alleviates lung injuries in sepsis-related ARDS by regulating NQO1-Nrf2 signaling pathway ( $\mathrm{Li}$ et al. 2015). miRNA-23a-5p can be used as a potential biomarker for early sepsis induced ARDS (Liu et al. 2016). Among various miRNA, in previous study, miR-146b mainly functions as a regulatory factor of inflammation and cancer (Huang et al. 2019), few studies are about its effect on ARDS (Yao et al. 2018).

In our study, a target relationship between miR-146b-3p and $\mathrm{PI} 3 \mathrm{~K} \gamma$ was found by bioinformatics prediction. Hence, we speculated that miR-146b-3p might repress PI3K/AKT signaling pathway by targeted down-regulation of PI3K $\gamma$ expression, thus improving ARDS in septic mice.

\section{Methods}

\section{Grouping and treating}

Ten of seventy 10 -week-old healthy male C57BL/6 mice of clean grade (purchased from the Laboratory Animal Center of Wenzhou Medical University), weighing $25 \pm 5$ g, were randomly selected as normal group $(n=10)$. The rest were used to construct septic mice models with ARDS (Sahetya et al. 2017). Briefly, mice were anesthetized by intraperitoneal administration of $40 \mathrm{mg} / \mathrm{kg}$ pentobarbital sodium. Their trachea and right internal jugular vein were then exposed and $50 \mathrm{~mL}$ of sterile LPS (escherichia coli LPS serotype 0111:B4) or phosphate buffered saline (PBS) were dripped into. A total of $3 \times 10^{7}$ PFU Ad-omentin or Ad- $\beta$-gal was injected into the internal jugular vein of mice 3 days before LPS or PBS administration. All mice in normal group were received PBS and they were survived. 18 mice received LPS died $24 \mathrm{~h}$ after administration. Forty two successfully modelled mice were divided into 6 groups: model group $(n=07$, without any treatment), negative control (NC) mimic group ( $n=07$, injected with NC mimic), miR-146b-3p mimic group ( $\mathrm{n}=07$, injected with miR-146b-3p mimic), si-NC group ( $\mathrm{n}=07$, injected with $\mathrm{PI} 3 \mathrm{~K} \gamma$ siRNA NC), si-PI3K $\gamma$ group $(\mathrm{n}=07$, injected with $\mathrm{PI} 3 \mathrm{~K} \gamma$ silencing plasmid), and miR-146b-3p mimic + oePI3K $\gamma$ group $(n=07$, injected with miR-146b-3p mimic + $\mathrm{PI} 3 \mathrm{~K} \gamma$ overexpression plasmid). RNA mimics and plasmids (Suzhou Jima Gene Co. Ltd., China) were intraperitoneally injected in mice once at the dose of $100 \mathrm{ng}$ on alternate days for 3 times. Mice in each group were anesthetized using 0.1 $\mathrm{mL} / 10 \mathrm{~g} 0.3 \%$ pentobarbital sodium solution after treatment. Then the eyeball was removed for blood collection and mice were died under anesthesia, and lung tissue specimens were reserved. This study was approved by the Ethics Committee of The First People's Hospital of Wenling (No. WF2019012).

\section{Dual-luciferase reporter system}

The binding site of miR-146b-3p and PI3K $\gamma$ was predicted by bioinformatics prediction website (www.targetscan.org), which was verified by dual-luciferase reporter system. PI3K wild type reporter plasmid (PGL3-PI3K $\gamma$ wt) and mutant reporter plasmid (at the binding site of miR-146b-3p and PI3K $\gamma$, PGL3-PI3K $\gamma$ mut) were constructed, respectively. Rellina plasmid and the two reporter plasmids were cotransfected into HEK293T cells with miR-146b-3p plasmid or NC plasmid, respectively. Dual-luciferase reporter assay was performed after $24 \mathrm{~h}$ cell transfection according to the instruction of dual-luciferase reporter kit (Promega, USA).

\section{qRT-PCR}

We extracted total RNA in the lung tissues from four mice in each group. By using Trizol (Item number 16096020, Thermo Fisher Scientific, New York, USA; Item number B1802, Harbin HaiGene Co. Ltd., China). Taq Man MicroRNA Assays Reverse Transcription Primer (Thermo Scientific, USA) was used to obtain cDNA. SYBR ${ }^{\circledR}$ Premix Ex Taq ${ }^{\text {TM }}$ II kit (Xingzhi Biotechnology Co. Ltd., China) was used to perform fluorescent quantitative PCR detection. The working solution included $25 \mu \mathrm{L}$ SYBR ${ }^{\circledR}$ Premix Ex Taq ${ }^{\mathrm{TM}} \mathrm{II}(2 \times), 2$ $\mu \mathrm{L}$ PCR forward primer, $2 \mu \mathrm{L}$ PCR reverse primer, $1 \mu \mathrm{L}$ ROX Reference Dye (50×), $4 \mu \mathrm{L}$ DNA templates and $16 \mu \mathrm{LddH}_{2} \mathrm{O}$. Primers were shown in Table 1. Fluorescent quantitative PCR was carried out by using ABIPRISM ${ }^{\circledR} 7300$ system (Prism ${ }^{\circledR 7300, ~ S h a n g h a i ~ K u n k e ~ I n s t r u m e n t ~ E q u i p m e n t ~ C o . ~}$ Ltd., China). The reaction condition was that 32 cycles of pre-denaturation at $95{ }^{\circ} \mathrm{C}$ for $10 \mathrm{~min}$, denaturation at $95{ }^{\circ} \mathrm{C}$ for $15 \mathrm{~s}$ and annealing at $60^{\circ} \mathrm{C}$ for $30 \mathrm{~s}$ followed by extending at $72{ }^{\circ} \mathrm{C}$ for $1 \mathrm{~min}$. miR-146b-3p took U6 as the internal reference and PI3K $\gamma$ used GAPDH as the internal reference. $2^{-\Delta \Delta \mathrm{Ct}}$ showed the relative expression level of a target gene.

\section{Western blot}

We extracted total protein in the lung tissues from four mice in each group by using RIPA lysis buffer containing PMSF (R0010, Solarbio). Protein concentration was determined using BCA kit (Thermo Fisher, USA), and the samples were mixed with loading buffer and heated on the boiling water bath for $10 \mathrm{~min}$. Then, $30 \mu \mathrm{g}$ of protein samples were added into sample well and electrophoresis was conducted at a constant voltage of $80 \mathrm{~V}$ for $2 \mathrm{~h}$. Proteins were transferred to PVDF membrane (ISEQ00010, Millipore, Billerica, MA, USA) at $110 \mathrm{~V}$ for $2 \mathrm{~h}$. The membrane was sealed with $5 \%$ skimmed milk powder at $4{ }^{\circ} \mathrm{C}$ for $2 \mathrm{~h}$ and washed once. The membrane was incubated with primary antibodies, rabbit antimouse PI3K $\gamma$ (ab140307, 1:1,000, Abcam, UK), AKT (ab179463, 1:10,000, Abcam, UK), AKT (phospho S473) 
Table 1 qRT-PCR primer sequence

\begin{tabular}{ll}
\hline Gene & Sequence \\
\hline miR-146b-3p & Forward: 5'-TGAGAACTGAATTCCATAGGCT-3' \\
& Reverse: 5'-GCACTGTCAGACCGAGACAAG-3' \\
U6 & Forward: 5'-CTCGCTTCGGCAGCACATATACT-3' \\
& Reverse: 5'-ACGCTTCACGAATTTGCGTGTC-3' \\
PI3K $\gamma$ & Forward: 5'-GACGAATTCAAATTATAACCTGGGAAATGGAG-3' \\
& Reverse: 5'-GCAAAGCTTCTTTTATGCCTGACATTTCTT-3' \\
GAPDH & Forward: 5'-CCAATGTGTCCGTCGTGGATCT-3' \\
& Reverse: 5'-GTTGAAGTCGCAGGAGACAACC-3' \\
\hline
\end{tabular}

(ab81283, 1:5,000, Abcam, UK), NLRP3 (ab232401, 1:1,000, Abcam, UK), ASC (ab155970, 1:1,000, Abcam, UK), Caspase-1 (ab238979, 1:1,000, Abcam, UK) and GAPDH (ab8226, 1:2,000, Abcam, UK) at $4{ }^{\circ} \mathrm{C}$ overnight and washed three times. The membrane was then incubated with horseradish peroxidase-labeled goat anti-rabbit IgG antibody (1:5,000, Beijing Zhongshan Biotechnology Co. Ltd., China) and washed three times. The membrane was developed by ECL fluorescence detection kit (Item Number BB-3501, Ameshame, UK) and exposed by a gel imager. Images were taken using Bio-Rad image analysis system (BIO-RAD, USA) and analyzed using ImageJ software. Relative protein expression = gray value of the corresponding protein band / gray value of GAPDH protein band.

\section{Wet weight/dry weight ratio}

The left lungs of three mice in per group were taken out by thoracotomy, and the blood on the lung surface was sucked dry by filter papers. Then wet weight was weighed out. After the left lung was baked in the oven at $80^{\circ} \mathrm{C}$ for $48 \mathrm{~h}$ to achieve constant weight, the dry weight was weighed out. Wet weight / dry weight (W/D) ratio and lung water content were calculated to reflect the severity of pulmonary edema. W/D ratio of the lung = wet weight of the lung / dry weight of the lung $\times$ $100 \%$.

\section{Hematoxylin and eosin (HE) staining}

The lung tissue from three mice in each group was routinely fixed, dehydrated, transparentized, embedded and sliced. Slices were transparentized with xylene, dehydrated in gradient alcohol and washed with distilled water for $1 \mathrm{~min}$. Slices were dewaxed routinely and differentiated in $0.5 \%$ hydrochloric acid alcohol differentiation solution for $10 \mathrm{~s}$, blued by rinsing with running water for $10 \mathrm{~min}$ and stained in eosin solution for $5 \mathrm{~min}$. Slices were dehydrated routinely, transparentized and sealed with neutral gum. Slices were observed under an optical microscope (XP-330, Shanghai Bingyu Optical Instrument Co. Ltd., Shanghai, China).

\section{ELISA}

Blood extracted from the eyeballs of all mice in per group was placed at room temperature first and at $4{ }^{\circ} \mathrm{C}$ overnight and centrifuged at 3,500 $\mathrm{xg}$. The supernatant was collected and cryopreserved at $-80^{\circ} \mathrm{C}$. Levels of serum IL-1 $\beta$ and IL-18 were detected by referring to the instructions of ELISA detection kits (IL-1 $\beta$ and IL-18 detection kits number: 69-59812, 69-21183, Wuhan MSKBio, China).

\section{Statistical analysis}

All data were analyzed by SPSS21.0 statistical software. The measurement data were expressed as mean \pm standard deviation. Comparison among groups was performed by one-way analysis of variance, and pairwise comparison of the mean among groups was carried out by Tukey posthoc test. $P<0.05$ indicated a statistically significant difference.

\section{Results}

\section{miR-146b-3p targeted the negative regulation of PI3KY gene}

The bioinformatics prediction website www.targetscan. org (http://www.targetscan.org/vert_72/) predicted that there was a specific binding site of miR-146b-3p and PI3K $\gamma$ (Fig. 1a), which was verified by dual-luciferase reporter system assay. The results showed that miR146b-3p mimic group had lower luciferase activity for co-transfecting with PGL3-PI3K $\gamma$ wt compared with NC mimic group $(P<0.05)$, and there was no significant difference in luciferase activity in cells transfected with PGL3-PI3K $\gamma$ mut $(P>0.05)$. Therefore, miR-146b-3p could target and negatively regulate the expression of PI3K gene (Fig. 1b). 
a

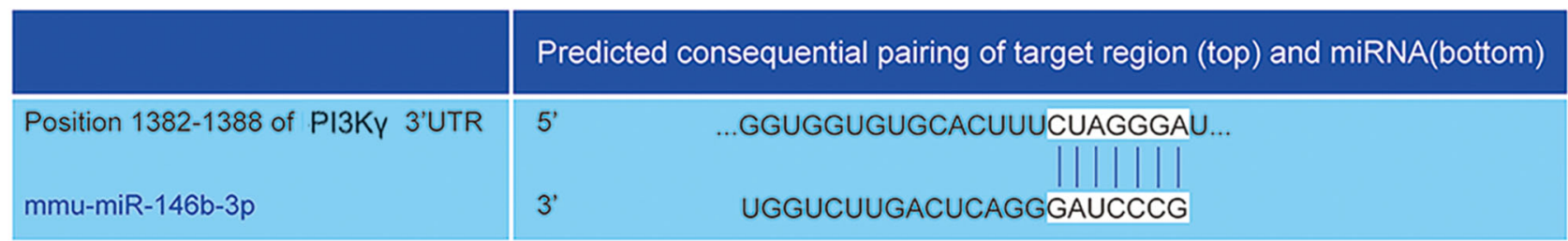

b

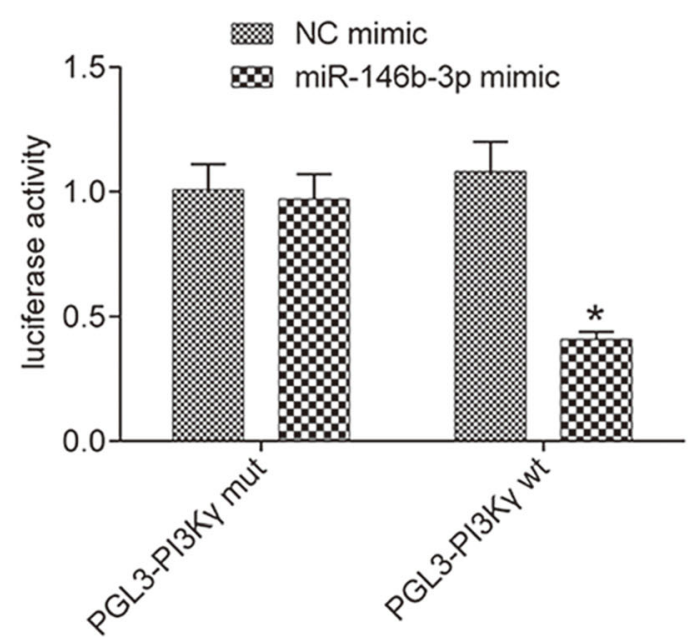

Fig. 1 miR-146b-3p inhibits PI3K/AKT signaling pathway in the lung tissues a Sequence of the binding site of miR-146b-3p 3'-UTR region and $\mathrm{PI} 3 \mathrm{~K} \gamma$. b Dual luciferase reporter system assay. Compared with the NC

\section{miR-146b-3p, PI3Ky, AKT, p-AKT expressions in the lung tissue}

We detected the expressions of PI3K/AKT signaling pathway in lung tissues in each group to further confirm the regulation relationship between miR-146b-3p and PI3K/AKT signaling pathway (Fig. 2). Compared with normal group, model mice had significantly lower miR-146b-3p expression and higher PI $3 \mathrm{~K} \gamma$ expression (both $P<0.05$ ). Compared with model mice, mice in miR-146b-3p mimic group and si-PI3K $\gamma$ group had significantly lower PI3K $\gamma$ and p-AKT protein expressions (both $P<0.05$ ). PI3K overexpression could weaken the effects of miR-146b-3p mimic.

\section{Pathological change of mouse lung}

Pathological change of mouse lung was detected by HE staining (Fig. 3). Mice in normal group had regular lung structure and no significant pathological injury. Mice in other groups had various degrees of inflammatory cell infiltration in the pulmonary alveoli and mesenchyme, effusion in the cavity, obvious thickening alveolar septum, partial collapsed alveoli, atelectasis, hyaline membrane, and damaged alveoli. Lung injuries in miR-146b-3p mimic group and si-PI3K $\gamma$ group were significantly improved than model group, $\mathrm{NC}$ mimic group, si-NC group, and miR-146b-3p mimic + oe-PI3K $\gamma$ group. mimic group, ${ }^{*} P<0.05$. wt: wild type, mut: mutant, NC: negative control, 3'-UTR: 3' untranslated region

\section{Wet weight / dry weight ratio of the lung}

W/D ratio of the lung was detected to evaluate lung edema (Fig. 4). Compared with normal group, model mice had significantly higher $\mathrm{W} / \mathrm{D}$ ratio $(P<0.05)$. Compared with model mice, mice in miR-146b-3p mimic group and si-PI3K $\gamma$ group had significantly lower W/ D ratio $(P<0.05)$. PI3K overexpression could weaken the effects of miR-146b-3p mimic.

\section{Levels of IL-1 $\beta$ and IL-18 in serum}

Levels of IL-1 $\beta$ and IL-18 in serum were detected by ELISA (Fig. 5). Levels of IL-1 $\beta$ and IL-18 were significantly higher in other groups than in normal group (both $P<0.05$ ). Compared with normal group, model mice had significantly higher levels of IL-1 $\beta$ and IL-18 (both $P<0.05$ ). Compared with model mice, mice in miR-146b-3p mimic group and siPI3K $\gamma$ group had significantly lower levels of IL-1 $\beta$ and IL18 (both $P<0.05$ ). PI3K overexpression could weaken the effects of miR-146b-3p mimic.

\section{NLRP3, ASC and Caspase-1 protein expressions of the lung}

Compared with normal group, model mice had significantly higher NLRP3, ASC and Caspase-1 protein expressions (all 
a

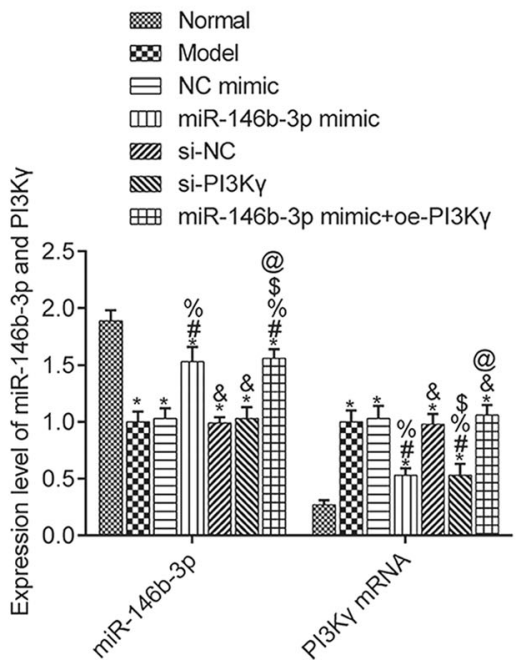

Fig. 2 miR-146b-3p inhibits Notch signaling pathway in the lung tissues a Expression levels of miR-146b-3p and PI3K $\gamma$. b Protein bands of PI3K $\gamma$, AKT and p-AKT. c Protein expressions of PI3K $\gamma$, AKT and pAKT. Compared with the normal group, ${ }^{*} P<0.05$; compared with the

$P<0.05$, Fig. 6). Compared with model mice, mice in miR$146 \mathrm{~b}-3 \mathrm{p}$ mimic group and si-PI3K $\gamma$ group had significantly lower NLRP3, ASC and Caspase-1 protein expressions (all $P<0.05$ ). PI3K overexpression could weaken the effects of miR-146b-3p mimic.

\section{Discussion}

Previous studies have reported that miR-146b-3p may be a blood potential biomarker for lung adenocarcinoma (Rani
C

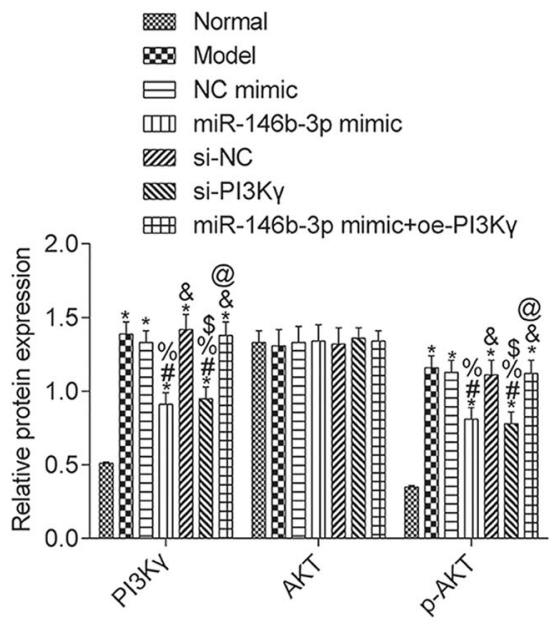

model group, ${ }^{\#} P<0.05$; compared with the $\mathrm{NC}$ mimic group, ${ }^{\%} \mathrm{P}<0.05$; compared with the miR-146b-3p mimic group, ${ }^{\&} P<0.05$; compared with the si-NC group, ${ }^{\$} P<0.05$; compared with the si-PI3K $\gamma$ group, ${ }^{\circledR} P<0.05$. NC: negative control

et al. 2013). For example, Sha et al. found that compared with patients with benign pulmonary nodules and healthy people, miR-146b-3p was significantly overexpressed in peripheral blood of patients with non-small cell lung cancer (Sha and Fan 2018). Thus, we speculated that miR-146b-3p may be involved in other lung diseases, such as sepsis-induced ARDS. But no related animal model or clinical researches have been published. In our study, down-regulated miR146b-3p was found in lung tissues of mice with sepsisinduced ARDS, indicating that miR-146b-3p may participates in the development of ARDS in sepsis mice. a
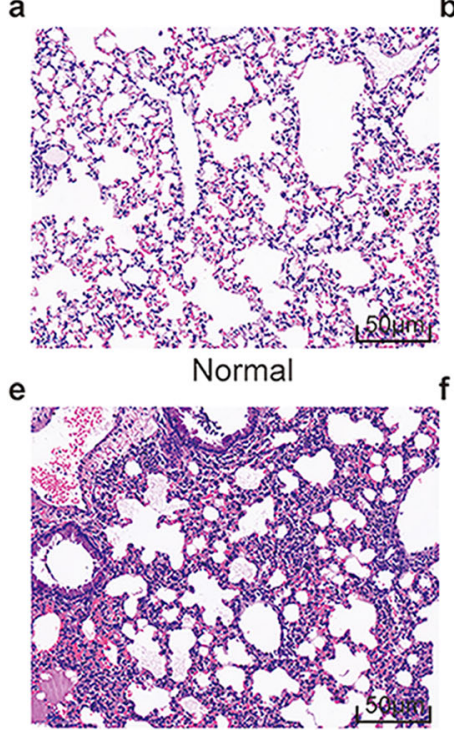

si-NC b

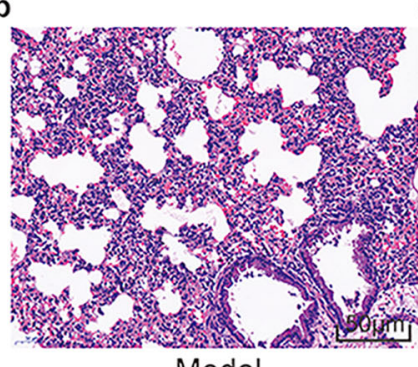

c

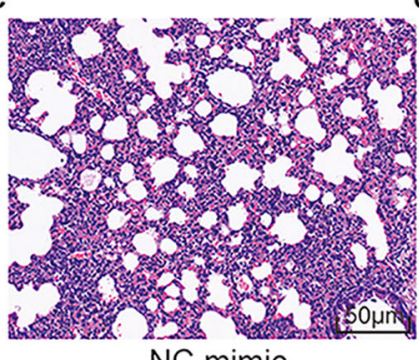

NC mimic

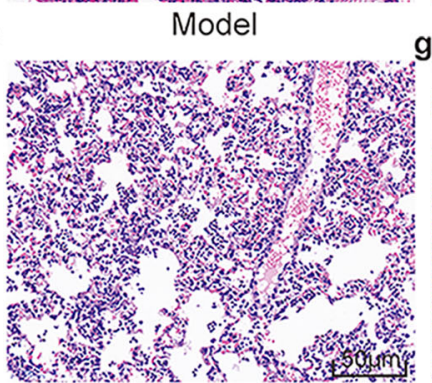

si-PI3Ky

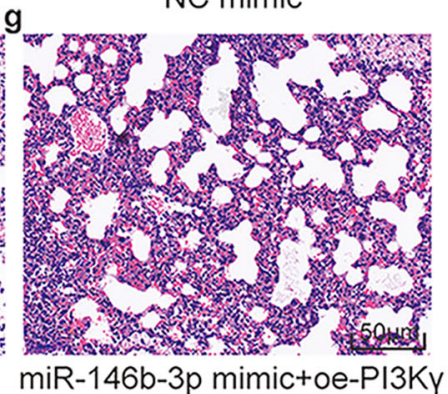

d

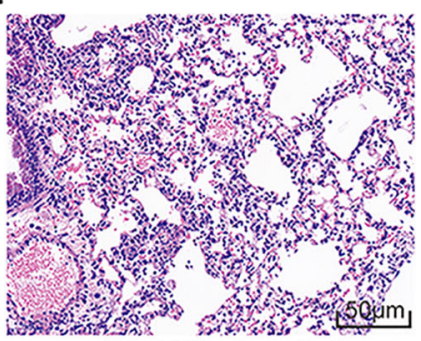

miR-146b-3p mimic

Fig. 3 Pathological change in the lung tissues $(200 \times)$ a Normal group. b Model group. $\mathbf{c}$ NC mimic group. d miR-146b-3p mimic group. e si-NC group. $\mathbf{f}$ si-PI3K $\gamma$ group. g miR-146b-3p mimic + oe-PI3K $\gamma$ group. NC: negative control 


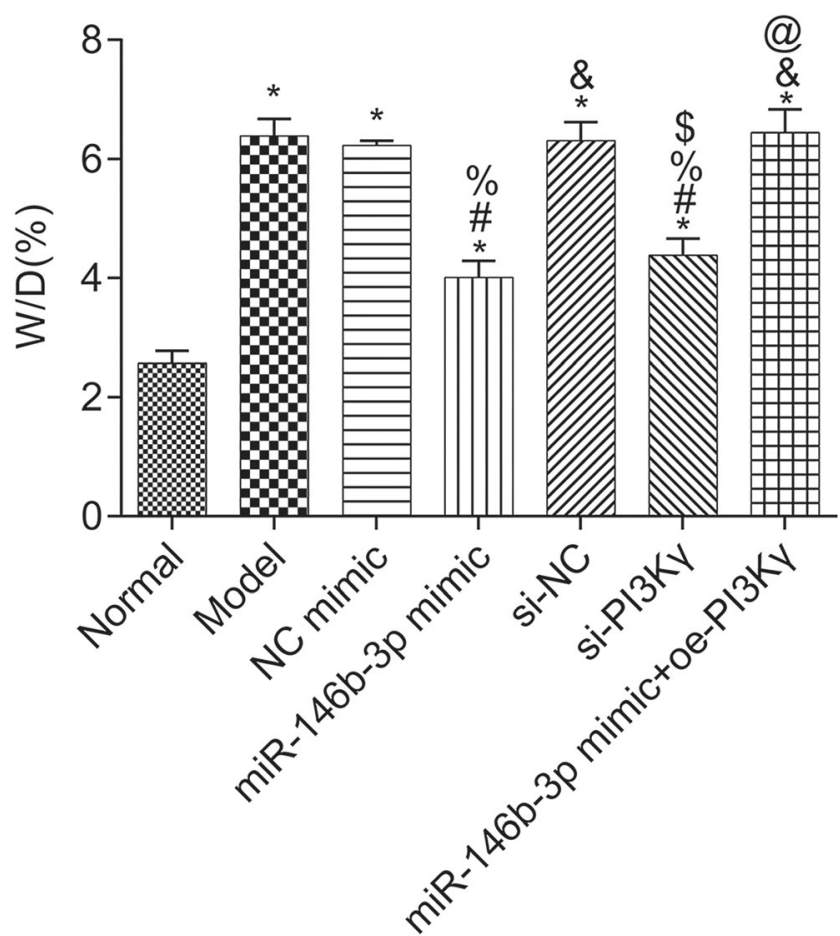

Fig. $4 \mathrm{~W} / \mathrm{D}$ ratio of the lung. Compared with the normal group, ${ }^{*} P<0.05$; compared with the model group, ${ }^{\#} P<0.05$; compared with the NC mimic group, ${ }^{\%} P<0.05$; compared with the miR-146b-3p mimic group, ${ }^{\&} P<0.05$; compared with the si-NC group, ${ }^{\$} P<0.05$; compared with the si-PI3K $\gamma$ group, ${ }^{\circledR} P<0.05$. W/D: Wet weight/dry weight, NC: negative control

a

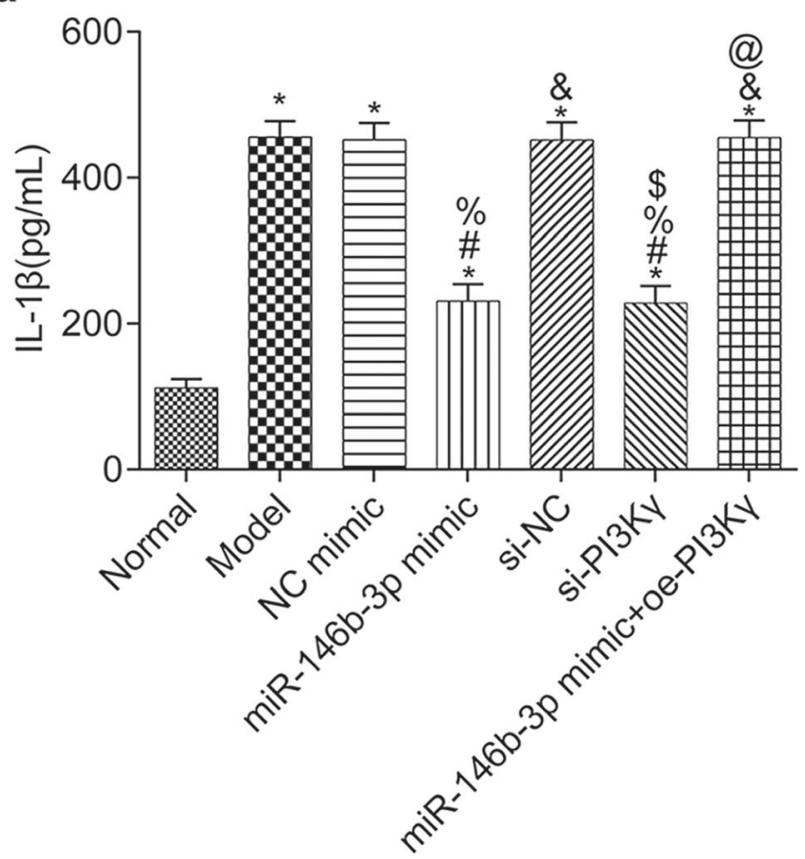

Fig. 5 Levels of IL-1 $\beta$ and IL-18 in serum a Level of IL-1 $\beta$. b Level of IL-18. Compared with the normal group, ${ }^{*} P<0.05$; compared with the model group, ${ }^{\#} P<0.05$; compared with the NC mimic group, ${ }^{\%} P<0.05$;
In order to further explore the mechanism of miR-146b-3p affecting ARDS, we used bioinformatic website to predict the possible target of miR-146b-3p and the result was verified by dual-luciferase reporter system assay. Finally, we found that miR-146b-3p could target and negatively regulate of PI3K gene expression. Literatures revealed the activated PI3K/ AKT signaling pathway in ARDS (Li et al. 2018b; Laffey and Matthay 2017). In this study, we also found that $t$ inhibition of PI3K/AKT signaling pathway could improve lung injuries caused by ARDS in septic mice. Therefore, we further speculated that miR-146b-3p may play a positive role in sepsis induced ARDS by inhibiting PI3K/AKT signaling pathway.

Mice with ARDS induce lung injury often had severe lung edema and abnormally elevated inflammatory response, increased lung cell apoptosis. Lung edema is a common inducement of ARDS, the degree of which can indicate the treatment efficiency (Saitoh et al. 2002). NLR3 inflammasome is a multi-protein complex composed of NLRR3, ASC and the inactive pro caspase-1, which is an important part of the inflammatory immune response. NLR3 amino terminal pyrin domain binds to ASC and then recruits pro-caspase-1 to form the inflammasome and then activate caspase- 1 to promote maturation and secretion of downstream IL-1 $\beta$ and IL-18 (Jiang et al. 2016; Madouri et al. 2015). Li et al. discovered that pirfenidone could inhibit the activation of NLRP3 inflammasome, which may be promising therapeutic strategy for ARDS (Li et al. 2018a, b, c). To further explore the

b

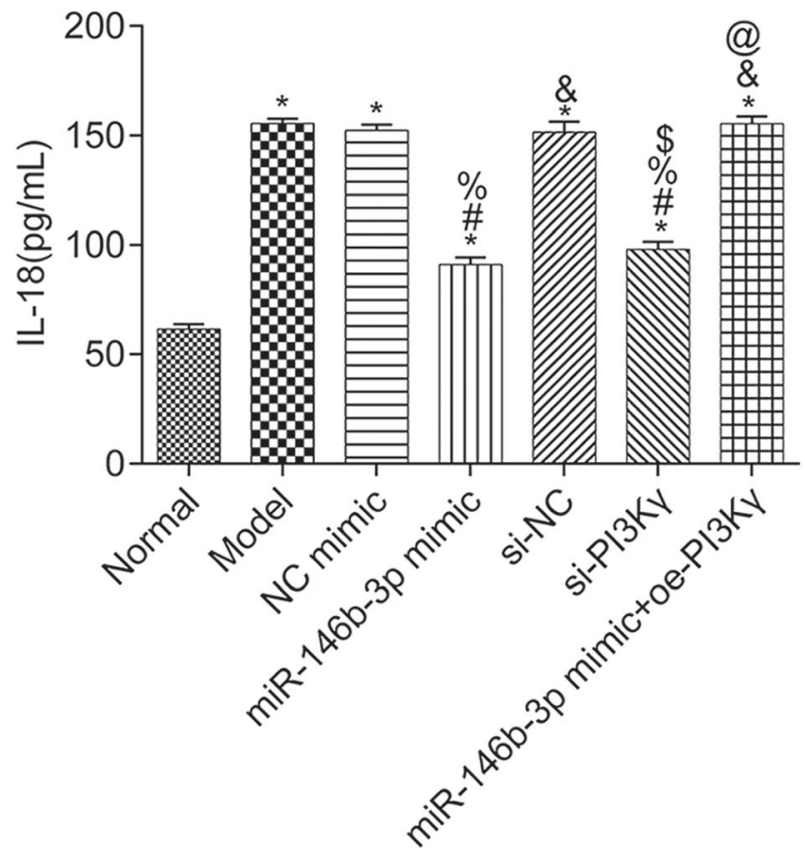

compared with the miR-146b-3p mimic group, ${ }^{\&} P<0.05$; compared with the si-NC group, ${ }^{\$} P<0.05$; compared with the si-PI3K $\gamma$ group, ${ }^{\circledR} P<0.05$. IL: interleukin, NC: negative control 
a

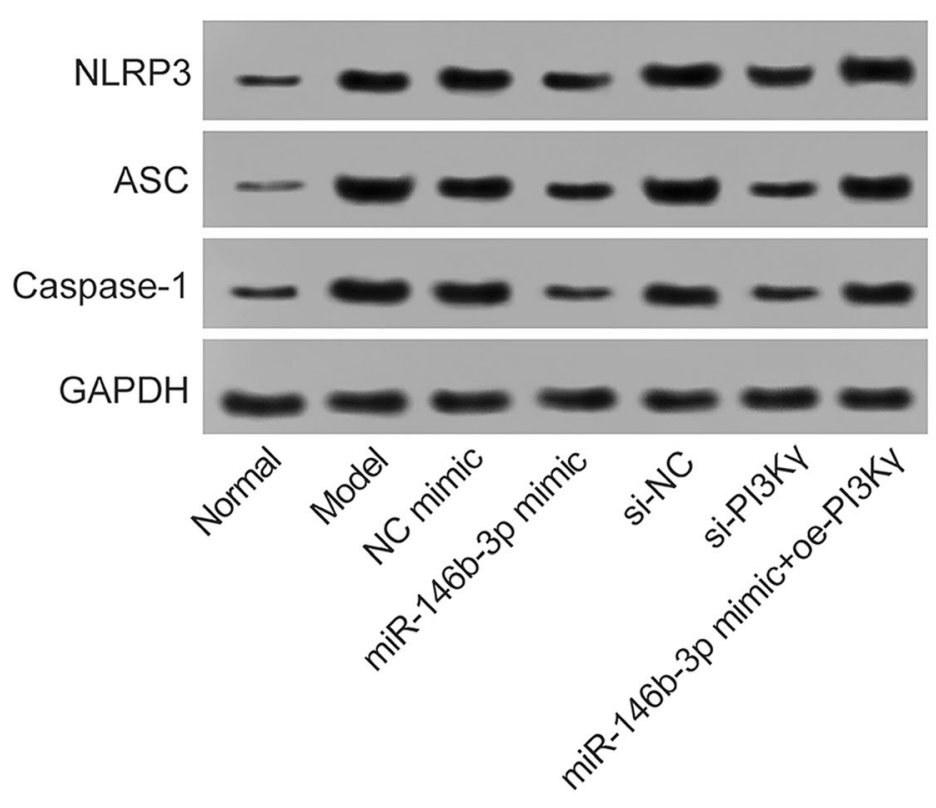

b
$\approx$ Normal
8 Model
$\boxminus \mathrm{NC}$ mimic
U1] miR-146b-3p mimic
WIA si-NC
si-PI3Ky
$\Xi$ miR-146b-3p mimic+oe-PI3Ky

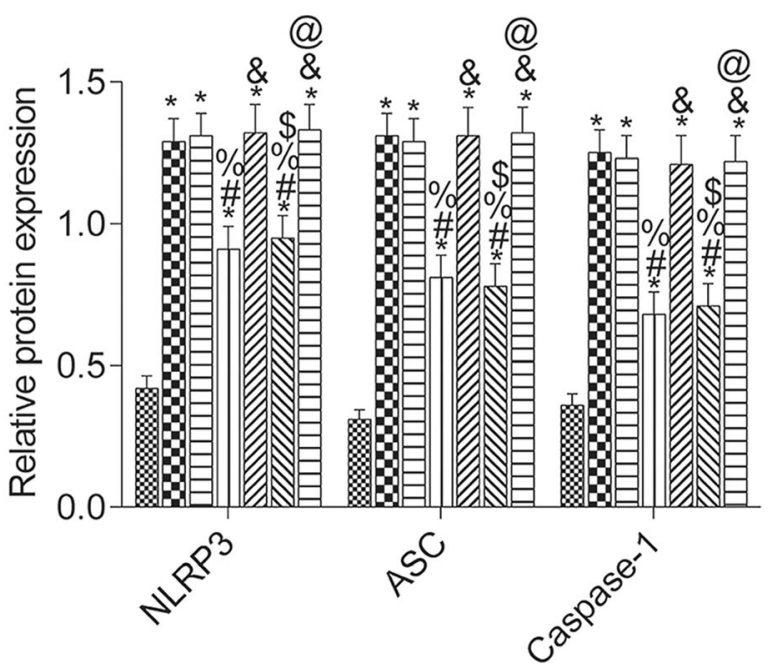

Fig. 6 NLRP3, ASC and Caspase-1 protein expressions in the lung tissues a Protein bands of NLRP3, ASC and Caspase-1. b NLRP3, ASC and Caspase-1 protein expressions. Compared with the normal group, ${ }^{*} P<0.05$; compared with the model group, ${ }^{\#} P<0.05$; compared with

upstream regulatory mechanism of miR-146b-3p and PI3K/ AKT signaling pathway, sepsis model mice were treated with miR-146b-3p mimic, miR-146b-3p inhibitor or PI3K $\gamma$ overexpression virus vectors. The results indicated miR-146b-3p inhibited PI3K/AKT signaling pathway by targeting and inhibiting $\mathrm{PI} 3 \mathrm{~K} \gamma$ gene expression, thereby down-regulating the expressions of inflammasome ASC, NLRP3 and Caspase- 1 and reducing IL- $1 \beta$ and IL-18 levels in serum, so as to alleviate lung injuries caused by ARDS in septic mice. Moreover, overexpression of PI3K reversed the protection of miR-146b-3p overexpression on lung injury. Our results were also consistent with previous reports on ARDS in septic mice (Afshari et al. 2010; Mittal and Sanyal 2012; Ding et al. 2017; Xie et al. 2019; Pham and Rubenfeld 2017).

However, the association between miR-146b-3p and ARDS in septic mice has not been fully explained to date; the molecular mechanism by which PI3K/AKT signaling pathway regulates inflammasome NLRP3, ASC and Caspase-1 has not been fully explored; the targeted regulatory network of miR-146b-3p in sepsis-induced ARDS mice is still unclear.

In conclusion, miR-146b-3p, by targeting PI3K $\gamma$ gene, mediates PI3K signaling pathway to inhibit the recovery of lung injury in model mice. We further clarify the development the NC mimic group, ${ }^{\%} P<0.05$; compared with the miR-146b-3p mimic group, ${ }^{\&} P<0.05$; compared with the si-NC group, ${ }^{\$} P<0.05$; compared with the si-PI3K $\gamma$ group, ${ }^{\circledR} P<0.05$. ASC: apoptosis-associated specklike protein caspase recruitment domain, $\mathrm{NC}$ : negative control

mechanism of ARDS in septic mice, which lays a theoretical foundation for the clinical treatment of ARDS.

Author contributions Conceptualization: Jun-Fen Zheng; Methodology: Jin-Qiang Zhu, Xiao-Hong Jin, Mei-Ping Dong; Formal analysis andinvestigation: Jin-Qiang Zhu, Xiao-Hong Jin; Writing - original draft preparation: Yao Liu, Jun-Fen Zheng; Writing - review andediting: Yao Liu, Jun-Fen Zheng. All authors read and approved the final manuscript.

\section{Compliance with ethical standards}

Conflict of interest The authors declare that they have no conflict of interest.

Ethics approval All applicable international, national, and/or institutional guidelines for the care and use of animals were followed. All procedures performed in studies involving animals were in accordance with the ethical standards of The First People's Hospital of Wenling (No. WF2019012).

\section{References}

Afshari A, Brok J, Moller AM, Wetterslev J (2010) Aerosolized prostacyclin for acute lung injury (ALI) and acute respiratory distress syndrome (ARDS). Cochrane Database Syst Rev (8):Cd007733 
Annane D, Sebille V, Bellissant E (2006) Effect of low doses of corticosteroids in septic shock patients with or without early acute respiratory distress syndrome. Crit Care Med 34(1):22-30

Chawla R, Mansuriya J, Modi N, Pandey A, Juneja D, Chawla A et al (2016) Acute respiratory distress syndrome: Predictors of noninvasive ventilation failure and intensive care unit mortality in clinical practice. J Crit Care 31(1):26-30

Ding Q, Liu GQ, Zeng YY, Zhu JJ, Liu ZY, Zhang X et al (2017) Role of IL-17 in LPS-induced acute lung injury: an in vivo study. Oncotarget 8(55):93704-93711

Huang W, Guo L, Zhao M, Zhang D, Xu H, Nie Q (2019) The Inhibition on MDFIC and PI3K/AKT Pathway Caused by miR-146b-3p Triggers Suppression of Myoblast Proliferation and Differentiation and Promotion of Apoptosis. Cells 8(7)

Ji S, Wang L (2019) mu-Opioid receptor signalling via PI3K/Akt pathway ameliorates lipopolysaccharide-induced acute respiratory distress syndrome. Exp Physiol 104(10):1555-1561

Jiang X, Zhong L, Sun D, Rong L (2016) Magnesium lithospermate B acts against dextran sodiumsulfate-induced ulcerative colitis by inhibiting activation of the NRLP3/ASC/Caspase-1 pathway. Environ Toxicol Pharmacol 41:72-77

Laffey JG, Matthay MA (2017) Fifty years of research in ARDS. Cellbased therapy for acute respiratory distress syndrome. Biology and potential therapeutic value. Am J Respir Crit Care Med 196(3):266273

Li Y et al (2018) Pirfenidone ameliorates lipopolysaccharide-induced pulmonary inflammation and fibrosis by blocking NLRP3 inflammasome\&nbsp;activation. Mol Immunol 99:134-144

Li X, Zhang J, Zhu X, Wang P, Wang X, Li D (2015) Progesterone reduces inflammation and apoptosis in neonatal rats with hypoxic ischemic brain damage through the PI3K/Akt pathway. Int J Clin Exp Med 8(5):8197-8203

Li D, Ren W, Jiang Z, Zhu L (2018a) Regulation of the NLRP3 inflammasome and macrophage pyroptosis by the p38 MAPK signaling pathway in a mouse model of acute lung injury. Mol Med Rep 18(5):4399-4409

Li W, Qi D, Chen L, Zhao Y, Deng W, Tang XM et al (2018b) Vaspin protects against lipopolysaccharide-induced acute respiratory distress syndrome in mice by inhibiting inflammation and protecting vascular endothelium via PI3K/Akt signal pathway. Nan Fang Yi Ke Da Xue Xue Bao 38(3):283-288

Liu S, Liu C, Wang Z, Huang J, Zeng Q (2016) microRNA-23a-5p acts as a potential biomarker for sepsis-induced acute respiratory distress syndrome in early stage. Cell Mol Biol (Noisy-le-grand) 62(2):3137

Madouri F et al (2015) Caspase-1 activation by NLRP3 inflammasome dampens IL-33-dependent house dust mite-induced allergic lung inflammation. J Mol Cell Biol 7:351-365

Mittal N, Sanyal SN (2012) Effect of exogenous surfactant on phosphatidylinositol 3-kinase-Akt pathway and peroxisome proliferator activated receptor-gamma during endotoxin induced acute respiratory distress syndrome. Mol Cell Biochem 361(1-2):135-141

Nystrom GJ, Lang CH (2008) Sepsis and AMPK Activation by AICAR Differentially Regulate FoxO-1, -3 and -4 mRNA in Striated Muscle. Int J Clin Exp Med 1(1):50-63

Park JI, Jung BH, Lee SG (2017) Veno-Arterial-Venous Hybrid Mode of Extracorporeal Membrane Oxygenation for Acute Respiratory Distress Syndrome Combined With Septic Shock in a Liver
Transplant Patient: A Case Report. Transplant Proc 49(5):11921195

Pham T, Rubenfeld GD (2017) Fifty Years of Research in ARDS. The Epidemiology of Acute Respiratory Distress Syndrome. A 50th Birthday Review. Am J Respir Crit Care Med 195(7):860-870

Qi D, Tang X, He J, Wang D, Zhao Y, Deng W et al (2016) Omentin protects against LPS-induced ARDS through suppressing pulmonary inflammation and promoting endothelial barrier via an Akt/ eNOS-dependent mechanism. Cell Death Dis 7(9):e2360

Rahmel T, Rump K, Adamzik M, Peters J, Frey UH (2018) Increased circulating microRNA-122 is associated with mortality and acute liver injury in the acute respiratory distress syndrome. BMC Anesthesiol 18(1):75

Rani S, Gately K, Crown J, O’Byrne K, O’Driscoll L (2013) Global analysis of serum microRNAs as potential biomarkers for lung adenocarcinoma. Cancer Biol Ther 14:1104-1112

Sahetya SK, Goligher EC, Brower RG (2017) Fifty Years of Research in ARDS. Setting Positive End-Expiratory Pressure in Acute Respiratory Distress Syndrome. Am J Respir Crit Care Med 195(11):1429-1438

Saitoh H, Masuda T, Zhang XY, Shimura S, Shirato K (2002) Effect of antisense oligonucleotides to nuclear factor-kappaB on the survival of LPS-induced ARDS in mouse. Exp Lung Res 28:219-231

Sha J, Fan LH (2018) Diagnostic value of miRNA-146b-3p in early nonsmall cell lung cancer. Tongji Daxue Xuebao, Yixueban 39(2)

Wang X, Wang X, Liu X, Wang X, Xu J, Hou S et al (2015) miR-15a/16 are upreuglated in the serum of neonatal sepsis patients and inhibit the LPS-induced inflammatory pathway. Int J Clin Exp Med 8(4): $5683-5690$

Wang S, Wang JY, Wang T, Hang CC, Shao R, Li CS (2017) A Novel Porcine Model of Septic Shock Induced by Acute Respiratory Distress Syndrome due to Methicillin-resistant Staphylococcus aureus. Chin Med J (Engl) 130(10):1226-1235

Wang S, Li Z, Chen Q, Wang L, Zheng J, Lin Z et al (2018) NF-kappaBInduced MicroRNA-211 Inhibits Interleukin-10 in macrophages of rats with Lipopolysaccharide-Induced acute respiratory distress syndrome. Cell Physiol Biochem 45(1):332-342

Xie T, Xu Q, Wan H, Xing S, Shang C, Gao Y et al (2019) Lipopolysaccharide promotes lung fibroblast proliferation through autophagy inhibition via activation of the PI3K-Akt-mTOR pathway. Lab Invest 99(5):625-633

Yanagi S, Tsubouchi H, Miura A, Matsumoto N, Nakazato M (2015) Breakdown of epithelial barrier integrity and overdrive activation of alveolar epithelial cells in the pathogenesis of acute respiratory distress syndrome and lung fibrosis. Biomed Res Int 2015:573210

Yao S, Xu J, Zhao K, Song P, Yan Q, Fan W et al (2018) Downregulation of HPGD by miR-146b-3p promotes cervical cancer cell proliferation, migration and anchorage-independent growth through activation of STAT3 and AKT pathways. Cell Death Dis 9(11):1055

Zheng X, Zhang W, Hu X (2018) Different concentrations of lipopolysaccharide regulate barrier function through the PI3K/Akt signalling pathway in human pulmonary microvascular endothelial cells. Sci Rep 8(1):9963

Publisher's note Springer Nature remains neutral with regard to jurisdictional claims in published maps and institutional affiliations. 